\title{
Prevention of symptomatic thrombosis with short term (low molecular weight) heparin in patients with rheumatoid arthritis after hip or knee replacement
}

\begin{abstract}
H A E M van Heereveld, R F J M Laan, F H J van den Hoogen, M C de Waal Malefijt,
\end{abstract} I R O Novakova, L B A van de Putte

\begin{abstract}
The need for prevention of venous thromboembolism (VTE) after total hip or knee replacement is obvious. However, the optimal regimen to achieve this remains to be defined. In patients with rheumatoid arthritis (RA) long term coumarins may not be necessary owing to the use of non-steroidal anti-inflammatory drugs (NSAIDs). 103 patients in whom 151 surgical procedures were performed (55 hip and 96 knee prostheses) were treated only with short term subcutaneous heparin. NSAIDs were used daily in $85 \%$ of the patients, and they were continued after hospital discharge. Only one patient developed symptomatic deep venous thrombosis during one year follow up. Bleeding complications were seen in $20 / 151$ (13\%) of the surgical procedures, all clinically judged as minor, and recovery was not delayed except in one case. Short term (low molecular weight) heparin appears to be an adequate, simple, and safe method for prevention of symptomatic VTE in patients with RA after knee or hip replacement, though further studies are necessary to confirm these preliminary findings.

(Ann Rheum Dis 2001;60:974-976)
\end{abstract}

Venous thromboembolism (VTE) is a common complication in patients after hip or knee replacement. Without prophylaxis the incidence of VTE is high: when phlebography is performed in all patients after surgery with or without clinical signs of thrombosis, deep venous thrombosis (DVT) is found in 40$80 \%,{ }^{1}$ of which almost $50 \%$ is localised above the level of the knee. When ventilation perfusion (V/Q) scanning is done in all patients undergoing a hip or knee replacement, pulmonary embolism is found in $20 \%$ of the cases, resulting in fatal embolism in $1-2 \%{ }^{2}$

The optimal regimen to prevent VTE remains to be defined. There are many strategies for prevention of venous thrombosis in these patients with high risk of developing thromboembolic complications, varying from mechanical prophylaxis, such as graded compression stockings, to diverse pharmacological agents. Vitamin K antagonists are difficult to manage and result in an increased bleeding risk. Other pharmacological agents such as low dose standard heparin ( $\mathrm{SH}$ ) provide incomplete protection. Low molecular weight heparin (LMWH) is currently the most commonly applied thromboprophylactic agent in Europe in patients undergoing major orthopaedic surgery. Although there is only limited evidence for the safety and efficacy of LMWH in patients with rheumatoid arthritis (RA), it is commonly used in this patient group.

In patients with RA, often using nonsteroidal anti-inflammatory drugs (NSAIDs), the risk of developing VTE is reported to be less than in patients with osteoarthritis who use NSAIDs less frequently. ${ }^{34}$ This might in part be because of the antiplatelet activity of NSAIDs. ${ }^{5}$ Long term treatment with oral anticoagulants, as is advocated for patients with osteoarthritis, may therefore not be necessary in patients with RA, and short term treatment with subcutaneous SH or LMWH may be sufficient to prevent VTE in these patients. To date, no data on the incidence of postdischarge thrombosis in patients with RA have been published. ${ }^{6}$ From 1987 onwards, patients with RA in our department were treated with short term subcutaneous SH and from October 1992 onwards with subcutaneous LMWH, only for as long as they were immobilised. In this paper the safety and efficacy of this regimen is reported.

\section{Patients and methods}

In 1987 a protocol for prophylaxis of VTE in patients with RA undergoing an uncomplicated hip or knee replacement was implemented. The patients were screened for important additional risk factors for venous thrombosis-namely, venous insufficiency, heart failure, obesity (Quetelet Index $30 \mathrm{~kg} / \mathrm{m}^{2}$ or more), age above 70 years, and a history of VTE. 
Thrombosis prophylaxis consisted of subcutaneous SH 5000 IU twice a day, starting two to six hours before surgery and was given for a minimum duration of seven days. Anticoagulation was discontinued as soon as the patient was adequately mobilised. The patients were mobilised according to a fixed regimen. From October 1992 onwards SH was replaced by nadroparin, an LMWH, because of ample reported evidence that $\mathrm{LMWH}$ is better than $\mathrm{SH}$ for prophylaxis of VTE. ${ }^{7}$ The dose of nadroparin was $7500 \mathrm{IC}-\mathrm{U}$ (2850 IU) once a day, or a higher dose once a day when the patient was obese.

Sonography, phlebography, and V/Q scanning were performed only in patients with clinical suspicion of thrombosis or embolism.

Also recorded were bleeding complications, such as wound haematoma or other signs of superficial bleeding, a decrease in haemoglobin of $20 \mathrm{~g} / 1$ or more compared with the first postoperative value or a need for transfusion of two or more units of blood, haemarthrosis, and haemodynamic instability.

The patients were seen every three months, the total duration of the follow up was one year. They were screened for signs and symptoms of pulmonary embolism, DVT, and chronic venous insufficiency.

To evaluate the efficacy and safety of this protocol all medical records of patients with RA who underwent a hip or knee replacement at the department of rheumatology of the University Hospital Nijmegen from January 1987 to April 1995 were studied.

\section{Results}

PATIENT CHARACTERISTICS

The total group comprised 103 patients (69 female, 34 male) who underwent 151 surgical procedures: 55 hip implantations and 96 knee implantations. The mean (SD) age of the patients was $62(11.2)$. Sixty five patients had only one joint replacement, 31 patients had two, four patients had three, and three patients had four surgical procedures in this period. There were no additional risk factors for VTE in 73 surgical procedures. Seven patients had a history of VTE, 25 patients were obese, 35 patients were 70 years or older, and six patients had a heart failure. During 51 surgical procedures one additional risk factor was present, 17 times two risk factors, and 10 times three risk factors. Most patients with RA used NSAIDs daily: of the total group of 151 surgical procedures, 130 times NSAIDs were used daily, three times they were used on demand, and 18 times no NSAIDs were used. There was no change in NSAID use at hospital discharge.

ANTITHROMBOTIC PROPHYLAXIS

SH was used in 107 surgical procedures. All patients were treated with $\mathrm{SH}$ in a dose of 5000 IU twice a day. The median duration of the $\mathrm{SH}$ administration was nine days (interquartile range 8-11 days). Nadroparin was used in 44 surgical procedures. The dose of nadroparin was 7500 IC-U once a day, except for seven obese patients, who received a higher dose
(10 000-20 000 IC-U a day). The median duration of nadroparin administration was 10 days (interquartile range 9-15 days).

\section{COMPLICATIONS}

All patients were available for follow up. The incidence of VTE in our population was 1 episode/103 patients/year (95\% confidence interval 0 to 4 ). This patient developed a phlebographically proven calf vein thrombosis on day 17 after the operation, seven days after $\mathrm{SH}$ was stopped. He was aged 60 and had venous insufficiency as additional risk factor for thrombosis. He used naproxen daily in a dose of $1250 \mathrm{mg} /$ day.

Bleeding complications were seen in 20/151 $(13 \%)$ surgical procedures. Fifteen times a wound haematoma developed, necessitating blood transfusion in six cases because of an associated decrease in haemoglobin concentration. In one instance a surgical decompression was performed because of pressure neuropathy of the peroneal nerve due to a haematoma. In none of the bleeding episodes were there signs of haemodynamic instability. The bleeding complications did not delay the patients' recovery except for the patient with the pressure neuropathy.

\section{Discussion}

VTE is a common complication in patients undergoing elective hip or knee surgery. Over the past 25 years several anticoagulant regimens have been shown to be effective, though no regimen offers complete protection.

Previous studies have suggested that the incidence of deep VTE in patients with RA undergoing hip or knee surgery is three to 10 times less than in patients with osteoarthritis. ${ }^{34}$ The explanation is likely to be multifactorial: patients with RA are on average younger, their body weight is lower, their haemoglobin is lower, and reports have been published indicating that their fibrinolytic activity is higher ${ }^{4}$; however, recent publications also seem to find the lower fibrinolytic activity. Another explanation for the lower rate of thromboembolic complications in patients with RA may be the frequent use of NSAIDs, with their antiplatelet activity. ${ }^{4}$ In 1994 an overview of randomised trials of antiplatelet treatment showed a significant, clinically important reduction in thrombosis and pulmonary embolism in patients undergoing surgery. The authors state that in orthopaedic surgery antiplatelet treatment can almost halve the odds of developing a DVT (from $53 \%$ to $37 \%$ ), and can reduce pulmonary embolism by more than half (from 5.4\% to $2.6 \%) .^{9}$ The interpretation of the results of this study, however, requires caution, because of inclusion of inadequate trials. A thromboprophylactic effect on VTE of NSAIDs other than aspirin has never been proved in clinical trials.

The results of our study are in accordance with the expected relatively low incidence of venous thrombosis in patients with RA, most of whom use NSAIDs daily. Only one patient in 151 surgical procedures developed symptoms of a deep vein thrombosis, while he was still in 
hospital, seven days after the heparin was discontinued. This event could also be classified as "post-discharge" thrombosis. No other case of in-hospital or post-discharge venous thrombosis was found, despite the short duration of heparin administration. This is in contrast with other studies, in which patients with osteoarthritis were predominantly studied, where post-discharge venous thrombosis is a common complication, occurring in $20 \%$ or more of the patients undergoing hip replacement. ${ }^{10}$ As far as we know this is the first study presenting the incidence of post-discharge venous thrombosis in patients with RA. We suggest that the use of NSAIDs, which were continued after discharge, may have a protective role in the development of late venous thrombosis. Recently, a new group of NSAIDs have been introduced, the COX-2 selective NSAIDs. These NSAIDs have no influence on the platelet aggregation because they do not influence thomboxane synthesis. This may be beneficial in the prevention of bleeding complications in surgical patients; the effect on the incidence of VTE is currently unknown.

In our study we focused on clinically symptomatic thromboembolic complications. Calf vein thrombosis remains asymptomatic in about $90 \%$ of the cases, ${ }^{11}$ so we may have missed the majority of cases. However, asymptomatic calf vein thrombosis seems to be clinically less important, because the incidence of pulmonary embolism or chronic venous insufficiency has been reported to be low. ${ }^{12}$ Recent publications, however, showed that asymptomatic DVT is associated with a significant risk of post-thrombotic syndrome. ${ }^{13}$ Proximal vein thrombosis is more frequently symptomatic than calf vein thrombosis, estimations varying from 20 to $60 \%{ }^{7}$ In proximal DVT the risk of pulmonary embolism is high-about $50 \%{ }^{14}$ There is also a considerable risk for chronic venous insufficiency: about $50 \%$ of patients develop chronic venous insufficiency five years after the thrombosis. ${ }^{15}$

As might be expected from the use of NSAIDs in combination with heparin, a relatively high rate of bleeding complications was found $(13 \%)$, whereas most investigations mention a bleeding risk of $1-10 \% .^{7}$ In our patients all bleeding events were clinically judged as mild, except in the patient with a pressure neuropathy of the peroneal nerve due to a haematoma.

We realise that this study has methodological limitations. It is a retrospective open study, and the sample size is not sufficiently large to exclude a clinically relevant thromboembolic risk (confidence interval 0 to 4 ). However, this study does suggest that short term subcutaneous $\mathrm{SH}$ or nadroparin is an adequate, simple, and safe method for the prevention of symptomatic thrombosis in patients with RA (using NSAIDs), undergoing hip or knee replacement. The absence of post-discharge thrombosis, especially, seems promising. We suggest that further prospective controlled studies are warranted to compare the safety and effectiveness of short term LMWH with extended thromboprophylaxis in these patients. In this study the role of NSAIDs in thromboprophylaxis should be highlighted.

1 Weinmann EE, Salzman EW. Deep vein thrombosis [review]. N Engl J Med 1994;331:1630-41.

2 Swayze OS, Nasser S, Roberson JR. Deep venous thrombosis in total hip arthroplasty. Orthop Clin North Am 1992; 23:359-64.

3 Sikorski JM, Hampson WG, Staddon GE. The natural history and etiology of deep vein thrombosis after total hip replacement. J Bone Joint Surg Br 1981;63:171-7.

4 Kelsey JL, Wood PHN, Charnley J. Prediction of thromboembolism following total hip replacement. Clin Orthop 1967; 114:247-58.

5 Doutremepuich F, Aguejouf O, Imbault P, Azougagh Oualane F, Doutremepuich C. Effect of low molecular heparin/non-steroidal antiinflammatory drugs association on an experimental thrombosis induced by laser. Thromb Res 1995;77:311-19.

6 Nurmohamed MT, Lems WF, Dijkmans BAC. Risk of postdischarge venous thromboembolism in patients with rheumatoid arthritis undergoing knee or hip arthroplasty. Is prolonged thromboprophylaxis warranted or dangerous? Ann Rheum Dis 1999;58;392-5.

7 Anderson DR, O'Brien BJ, Levine MN, Roberts R, Wells PS, Hirsh J. Efficacy and cost of low-molecular-weight heparin compared with standard heparin for the prevention of deep vein thrombosis after total hip arthroplasty. Ann Intern Med 1993;119:1105-12.

8 White RA. Preoperative evaluation of patients with rheumatoid arthritis. Semin Arthritis Rheum 1985;14:287-99.

9 Antiplatelet trialists collaboration. Collaborative overview of randomised controlled trials of antiplatelet drug therapyIII: reduction in venous thrombosis and pulmonary embolism by antiplatelet prophylaxis among surgical and medical patients. BMJ 1994;235-46.

10 Arcelus JI, Caprini JA, Traverso CI. Venous thromboembolism after hospital discharge. Semin Thromb Haemost 1993;19(suppl):142-6.

11 Kakkar VV, Cohen AT, Edmondson RA, Phillips MJ, Cooper DJ, Das SK, et al. Low molecular weight versus standard heparin for prevention of venous thromboembolism after major abdominal surgey. Lancet 1993;341:25965.

12 Philbrick JT, Becker BM. Calf deep venous thrombosis. A wolf in sheeps clothing? Arch Intern Med 1988;148: 2131-8

13 Siragusa S, Beltrametti C, Barone M, Piovella F. Clinical course and incidence of post-phlebitic syndrome after profound asymptomatic deep vein thrombosis. Results of a transverse epidemiologic study. Minerva Cardioangiol 1997;45:57-66.

14 Moser KM, Lemoine JR. Is embolic risk conditioned by location of deep venous thrombosis? Ann Intern Med 1981;94:439-44.

15 Francis CW, Ricotta JW, Evarts CM, Marder VJ. Long-term clinical observations and venous functional abnormalities after asymptomatic venous thrombosis following total hip or knee arthroplasty. Clin Orthop 1988;232:271-8. 